\title{
Philosophiques
}

\section{Le réel de la philosophie et la pratique de la connaissance Foucault, Platon, Gueroult}

\section{Julien Panais}

Volume 47, numéro 1, printemps 2020

URI : https://id.erudit.org/iderudit/1070255ar

DOI : https://doi.org/10.7202/1070255ar

Aller au sommaire du numéro

\section{Éditeur(s)}

Société de philosophie du Québec

\section{ISSN}

0316-2923 (imprimé)

1492-1391 (numérique)

Découvrir la revue

\section{Citer cet article}

Panais, J. (2020). Le réel de la philosophie et la pratique de la connaissance : Foucault, Platon, Gueroult. Philosophiques, 47(1), 165-187.

https://doi.org/10.7202/1070255ar

\section{Résumé de l'article}

Lors d'un cours portant sur la Lettre VII de Platon, Michel Foucault introduit l'expression de "réel de la philosophie ». Pour les différents commentateurs de cette leçon, cette formule se rapporte aux actions politiquement efficaces que le philosophe doit effectuer pour insérer la philosophie dans la réalité. Selon nous et contrairement à cette interprétation, la philosophie possède déjà pour Foucault les modalités de sa propre effectivité avant même d'être efficace politiquement. La surestimation de la dimension politique conduit d'autre part à sous-estimer les pratiques de connaissance propre à la philosophie, d'autant que selon nous Foucault se saisit de la notion de "réel philosophique " de Martial Gueroult pour sa propre élaboration du « réel de la philosophie ». Déterminer les modalités du réel de la philosophie selon Foucault, c'est ce à quoi nous nous attachons dans cet article. 


\title{
Le réel de la philosophie et la pratique de la connaissance
}

Foucault, Platon, Gueroult

\author{
JULIEN PANAIS
}

Université de Lille

julien.panais@gmail.com

\begin{abstract}
RÉSUMÉ. - Lors d'un cours portant sur la Lettre VII de Platon, Michel Foucault introduit l'expression de «réel de la philosophie». Pour les différents commentateurs de cette leçon, cette formule se rapporte aux actions politiquement efficaces que le philosophe doit effectuer pour insérer la philosophie dans la réalité. Selon nous et contrairement à cette interprétation, la philosophie possède déjà pour Foucault les modalités de sa propre effectivité avant même d'être efficace politiquement. La surestimation de la dimension politique conduit d'autre part à sous-estimer les pratiques de connaissance propre à la philosophie, d'autant que selon nous Foucault se saisit de la notion de «réel philosophique» de Martial Gueroult pour sa propre élaboration du «réel de la philosophie». Déterminer les modalités du réel de la philosophie selon Foucault, c'est ce à quoi nous nous attachons dans cet article.
\end{abstract}

ABSTRACT. - During a lesson on Plato's Letter VII, Michel Foucault introduces the notion of «reality of philosophy». This notion, for some readers of this Foucault's lesson, relates to political efficient acts which philosopher must carry out to insert philosophy in reality. Rather, we believe that philosophy has already for Foucault conditions of its own effectiveness even before political efficiency. This overstatement of political sphere leads on the other side to underestimate knowledge practices of philosophy, especially as we believe that Foucault seizes the «philosophical reality» notion of Martial Gueroult to elaborate his own idea of philosophy reality. In this article, we try to figure out the philosophy reality conditions according to Foucault.

L'expression de «réel de la philosophie " prononcée par Foucault dans son cours de 1983 renvoie, dit-il, à un problème " familier et mal connu ", et à l'un des principes permanents de la philosophie depuis deux millénaires et demi au moins, celui d'une pratique de dire-vrai à l'égard du pouvoir ${ }^{1}$. Elle apparaît dans son étude de la Lettre VII de Platon pour commenter la «sorte d'obligation interne » de la philosophie qui constitue également sa tâche même:

Quand il s'inquiète de n'être que logos, quand il veut, plutôt que d'être simplement logos, toucher à la tâche même (à l'ergon) [de la philosophie], il me semble que Platon soulève une question que l'on pourrait appeler celle du réel

1. Je souhaite ici exprimer toute ma gratitude à Thomas Bénatouil qui a accompagné avec sollicitude mes recherches dans le cadre d'un doctorat, et qui a grandement participé à la finalisation de cet article par ses indications, objections et précisions. Je remercie également Bernard Sève qui m’a suggéré le rapprochement entre le réel de la philosophie chez Foucault et le réel philosophique de Gueroult. 
de la philosophie. Qu'est-ce que le réel de la philosophie? Où peut-on trouver le réel de la philosophie ${ }^{2}$ ?

Autrement dit, en montrant ce en quoi consiste l'ergon de la philosophie dans la Lettre VII, Foucault souhaite découvrir comment selon Platon la philosophie "peut devenir une activité réelle dans le réel » $(G S A, 239)$.

La tâche propre de la philosophie amena Platon jusqu'en Sicile en vue d'éduquer Denys, son jeune tyran. Par amitié pour Dion, un proche de l'autocrate qui lui demandait d'intervenir, et parce que jamais les circonstances (le kairos) n'ont paru aussi favorables pour réaliser "l'union dans les mêmes hommes, de la philosophie et de la conduite des grandes cités ${ }^{3}$ ", Platon s'embarqua pour Syracuse. S'il avait refusé, il aurait eu l'impression de n'être que "verbe-creux» (logos) et de paraître comme tel; or, il voulait se montrer aussi capable de mettre la main à l'action (à l'ergon). On retrouve ici dans cette lettre, explique Foucault, l'opposition très classique dans le vocabulaire grec entre logô et ergô — « en parole et en réalité, en discours et en acte»-; cette distinction reprise par Platon signifierait qu'il incombe au philosophe de n'être pas seulement l'auteur de La République, celui qui énonce ce qu'est la cité idéale, mais de remplir aussi la tâche du conseiller politique qui élabore, à travers l'âme du Prince, la rationalité de la conduite réelle de la cité ( $G S A, 202)$.

\section{Le réel de la philosophie comme rapport entre théorie et pratique}

Les commentateurs de la leçon de Foucault sur la Lettre VII s'accordent d'abord pour dire que Foucault s'approprie l'idée selon laquelle «l'activité philosophique ne doit pas se cantonner au seul discours, mais se mettre à l'épreuve des pratiques, des conflits et des faits ${ }^{4}$ ». Selon Hervé Oulc'hen, pour que la philosophie se suscite comme ergon, c'est-à-dire comme tâche pratique, «elle doit sortir de sa tour d'ivoire» en s'actualisant dans la conjoncture politique par un passage à l'acte ${ }^{5}$. Daniele Lorenzini fait de la question du réel de la philosophie celle de "l'efficacité concrète de son discours » : il ne suffit plus au philosophe de penser la cité idéale, il doit essayer de «la traduire dans la réalité ${ }^{*}$. Le philosophe isolé dans sa tour d'ivoire

2. Michel Foucault, Le gouvernement de soi et des autres, Paris, Seuil-Gallimard, 2008, p. 209, 2I I-2I2, désormais cité GSA.

3. Platon, Lettre VII, dans Euvres complètes, t. XIII-I, Lettres, trad. J. Souilhé, Paris, Les Belles Lettres, I960, p. 3I, 328b.

4. Frédéric Gros, "Situation du cours", dans GSA, p. 354.

5. Hervé Oulc'hen, L'intelligibilité de la pratique: Entre Foucault et Sartre, thèse de doctorat, Université Michel de Montaigne-Bordeaux III, 20I3, p. I62. Voir également sur ce point Marc Djaballah, "Le réel de la philosophie. Foucault et la critique ontologique ", Symposium, $\mathrm{n}^{\circ}$ I7-2, 20I3, p. II 5-I39.

6. Daniele Lorenzini, "La vie comme "réel" de la philosophie. Cavell, Foucault, Hadot et les techniques de l'ordinaire", dans Sandra Laugier (dir.), La voix et la vertu, Paris, PUF, 20I0, p. 469-487. 
— image constante du philosophe à l'état de nature dans ce type de lecture - ne doit plus se satisfaire de la théorie mais tenter de l'actualiser en pratique dans la réalité, c'est-à-dire dans le champ politique pris en un sens large; celui-ci pour Lorenzini ne peut pas en effet être séparé de façon $a$ priori de l'éthique: «si la cible principale du gouvernement des êtres humains est leur vie quotidienne» alors «tout partage entre politique et éthique s'avère une pure fiction ou une illusion de perspective", et il "devient par conséquent possible de concevoir l'éthique comme une pratique (politique)

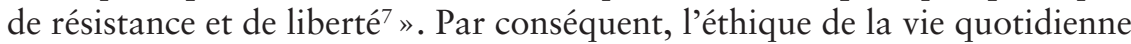
est selon lui directement politique.

Pourtant, selon Lorenzini, si la vie quotidienne des êtres humains est immédiatement politique, il n'en va pas de même pour le philosophe, lequel est incapable d'une pratique éthico-politique, à l'exception du philosophe cynique: "Les cyniques se présentent comme les seuls qui pratiquent réellement la philosophie ", car seuls ils appliquent "concrètement à la vie quotidienne" ce que tous les autres philosophes admettent "en théorie» mais qu'ils maintiennent «seulement dans l'élément du $\operatorname{logos}^{8}$ ». Le logos est assimilé à la théorie "abstraite", tandis que l'ergon constitue la pratique politique du philosophe qui se veut, en plus, efficace par sa mise en œuvre dans la vie quotidienne. Le réel pris dans le sens d'une efficacité pratique, c'est-àdire politique, devrait logiquement mener Lorenzini à un constat sans appel pour la philosophie, qu'il s'abstient cependant de formuler: depuis l'Antiquité, à l'exception des cyniques, la philosophie n'a pas «réellement " existé.

Dans cet article, nous voudrions proposer une tout autre interprétation de cette « réalisation» de la philosophie. Selon nous, à travers la lecture de la Lettre VII et l'étude de la philosophie antique, il s'agit pour Foucault d'envisager la philosophie moderne en sa réalité, plutôt que de chercher comment une philosophie pourrait descendre du ciel de la théorie dans le réel. Car pour lui la philosophie moderne s'est bien "effectivement inscrite dans le réel et dans l'histoire, ou plutôt dans ce réel qu'est notre histoire" (GSA, 32I). Le réel de la philosophie n'est pas à comprendre selon nous à travers la modalité de l'efficacité, mais à travers celle de l'effectivité. Pour le démontrer, il faut comprendre que Foucault hérite du problème du réel de la philosophie de Martial Gueroult, puis examiner comment il le pose dans le cadre de l'étude de la philosophie impériale ou platonicienne.

\section{La parenté de l'archéologie avec la dianoématique}

Lorsqu'il prononce l'expression de "réel de la philosophie », celle-ci paraît venir de loin et d'ailleurs. Foucault répète que c'est un problème "familier et mal connu» soulevé par Platon, «une question qui est à la fois très fami-

7. Daniele Lorenzini, Éthique et politique de soi. Foucault, Hadot, Cavell et les pratiques de l'ordinaire, Paris, Vrin, 20I 5, p. I3-I4.

8. Daniele Lorenzini, «La vie comme "réel” de la philosophie ", p. 477 (l'auteur souligne). 
lière, très évidente, transparente, et en même temps très mal connue » (GSA, 209, 207). Pourtant, jamais au cours des leçons il n'explique en quoi consiste cette évidence ignorée. Le « réel de la philosophie » n'est-elle pas une expression empruntée à Martial Gueroult et sa notion de "réel philosophique » développée dans Philosophie de l'histoire de la philosophie? C'est l'hypothèse que nous formulons ici, et les similitudes observées en premier lieu entre l'archéologie foucaldienne et la dianoématique gueroultienne nous engagent à l'explorer.

Commençons par remarquer la mention de Gueroult dans l'introduction au seul livre de Foucault consacré à une méthodologie générale, L'Archéologie du savoir publié en I969. Dans l'histoire de la philosophie, Martial Gueroult s'est détaché d'une analyse par «description des influences, des traditions, des continuités culturelles » au profit d'une analyse des unités architectoniques des systèmes qui effectue la description «des cohérences internes, des axiomes, des chaînes déductives, des compatibilités ${ }^{9}$ ». Foucault reprend l'idée d'unité architectonique dans son archéologie sous le terme d'archive: l'archive, c'est le système qui, «dans l'épaisseur des pratiques discursives", instaure "les énoncés comme des événements [...] et des choses ", c'est le champ de leurs conditions d'émergence et la loi de leur coexistence. L'archive, c'est "la loi de ce qui peut être dit ", c'est «la condition de réalité pour des énoncés" $(A S$, I 74-I77).

Dans cette mesure, la définition de l'archéologie foucaldienne est identique à celle de la dianoématique dans le domaine de l'histoire de la philosophie. Qu'est-ce que la dianoématique de Gueroult? Une discipline qui a pour tâche, premièrement, "de rendre compte de faits donnés: l'existence de métaphysiques que nous trouvons déjà constituées dans l'histoire» (caractère positif de la dianoématique); et, deuxièmement, «de savoir comment est possible un tel donné métaphysique, à sa façon, objectivement valable» (caractère transcendantal). Le transcendantal caractérise ici «la loi de ce qui peut être dit» dans l'ordre des déductions et des cohérences internes. En résumé, il s'agit de savoir "comment est possible "l'expérience philosophique" présente dans l'histoire ${ }^{10}$ ». De même, l'archéologie foucaldienne présente ces deux dimensions, positive et transcendantale, qui se recoupent dans l'a priori historique. Elle est positive dans la mesure où l'a priori rend compte "d'une histoire qui est donnée, puisque c'est celle des choses effectivement dites» - et non des choses possiblement données à l'expérience - traitées dans le jeu de leur instance $(A S, \mathrm{I} 74,39)$; transcendantale, puisqu'il s'agit de trouver, dans l'histoire des choses effectivement dites, l'a priori, condition de leur réalité en même temps que de leur émer-

9. Michel Foucault, L’Archéologie du savoir, Paris, Gallimard, «Tel», I969, p. 9-I2, ouvrage désormais cité $A S$.

10. Martial Gueroult, Dianoématique, livre II, Philosophie de l'histoire de la philosophie, Paris, Aubier Montaigne, I979, p. 65-66 (l'auteur souligne), désormais cité PHP. 
gence, défini comme «ensemble des règles qui caractérisent une pratique discursive " $(A S, \mathrm{I} 75)$ - et qui permet la venue au jour des choses dites. Pour l'archéologie foucaldienne comme pour la dianoématique, les conditions de possibilité sont des conditions de réalité. On constate ainsi la proximité des deux "disciplines"; ce qui amène à penser comme plus que probable la grande attention que Foucault a dû prêter à la publication posthume de Philosophie de l'histoire de la philosophie en 1979.

Dans son cours de I98I, Foucault reprend le constat déjà énoncé dans L'archéologie du savoir: la pratique discursive ne sert pas seulement à dénommer. Il écrivait à la fin des années 1960: "Certes, les discours sont faits de signes; mais ce qu'ils font, c'est plus que d'utiliser ces signes pour désigner des choses. [...] C'est ce "plus" qu'il faut faire apparaître et qu'il faut décrire " $(A S, 7 \mathrm{I})$. De son côté, Gueroult écrit dans son manuscrit: "Si la vérité est une copie, elle ne sert à rien, car elle n'ajoute rien au réel. Si elle ajoute quelque chose, alors elle ne peut pas connaître la réalité puisqu'elle la transforme" $(P H P, 79)$. Foucault reprend en I98 I l'énoncé de L'archéologie du savoir, mais cette fois-ci dans le vocabulaire de Gueroult: "Pourquoi y a-t-il, en plus du réel, du vrai? ? Pourquoi ce «jeu de véridiction qui vient s'ajouter au réel et qui le transmue, qui le transforme » $(S V, 240)$ ? Car, si le discours ne fait que refléter le réel, n'apparait-il pas comme "discours en $\operatorname{trop}^{11} »(S V, 235)$ ?

Il est du dessein de la science, selon Gueroult, de livrer la «vérité du monde", c'est-à-dire de fournir une image authentique de la réalité par adaequatio rei et intellectus. Mais qu'une telle finalité soit assignée à la philosophie pose un problème, car en ce cas elle ne se distingue pas de la science et n'a plus ni "mission propre» ni autonomie, quand bien même elle aurait pour objet, non pas le monde, mais l'essence de la conscience par laquelle toute vérité est fondée; "la vérité de l'esprit ne saurait être saisie, en l'espèce, qu'en fonction de la vérité scientifique ». Celle-ci variant selon le progrès de la science, le propre de la philosophie serait alors à chercher dans la positivité scientifique ( $P H P$, I6-I7). La «mission intellective» peut bien être ajustée à la science, en revanche, elle ne peut expliquer la raison d'être de la philosophie. Foucault reprend le raisonnement en en radicalisant la position: "La même question, la même difficulté se pose et doit se poser à propos de n'importe quel discours. » D'abord pour les discours moraux et prescriptifs qui prétendent "faire exister ce qui n'existe pas encore", mais même pour ceux qui "prétendent dire ce qui est»: "Jamais le fait que le ciel est bleu ne pourra rendre compte du fait que je dis que le ciel est bleu» ( $S V$, 224). Le fait que je décrive le réel, que je dise la vérité du réel, ne constitue jamais la raison pour laquelle je le dis: "Que telle proposition soit vraie, on peut l'établir en établissant que les choses sont telles que l'établit la propo-

11. Michel Foucault, Subjectivité et vérité, Paris, Gallimard-Seuil, 20I4, p. 240 et 235, désormais cité $S V$. 
sition» - reformulation tautologique par Foucault de la vérité comme adaequatio rei et intellectus -, "mais ce n'est pas parce que les choses sont telles, que le discours à l'intérieur duquel se trouve la proposition va exister comme réel » $(S V, 239)$.

Foucault s'intéresse ici au réel du discours qui tente de dire la réalité des choses. L'adéquation entre l'énoncé et la chose ne rend pas en soi l'énoncé digne d'intérêt (digne d'être dit et conservé); elle ne constitue pas la raison de «l'enracinement $[\mathrm{du}]$ jeu de la vérité dans le réel ». C'est pourquoi l'existence d'un discours à prétention de vérité «n'est jamais impliquée par la réalité des choses dont il parle» $(S V$, 224-225). De la même façon, Gueroult soutient que la pensée philosophante n'est pas engendrée par la réalité de la chose à expliquer, car l'intellection de l'objet, pour la pensée philosophante, ne fournit pas sa justification (PHP, 94-95). D'abord parce que la philosophie n'aurait pas de mission propre qui la distingue de la science, mais aussi parce que si tel était le cas pour Gueroult, les diverses doctrines philosophiques, privées de leur réalité et de leur valeur, n'auraient plus d'intérêt pour l'historien de la philosophie. Envisagée sous l'intellection scientifique, où donc en effet l'historien trouverait-il les "vérités prouvées» de la philosophie parmi toutes les doctrines contradictoires entre elles $(P H P, 23)$ ?

\section{Le réel philosophique de la dianoématique et l'autonomie de la volonté}

Nous avons vu la proximité de la méthode (positive et transcendantale) de Gueroult avec celle de Foucault, et du problème qui leur est commun; nous allons maintenant nous concentrer sur la dianoématique pour observer ensuite ce que Foucault peut lui emprunter.

La dianoématique de Gueroult se fonde sur un premier postulat, condition de possibilité de toute l'entreprise: la réalité de chaque doctrine philosophique $(P H P, 66)$. En un premier sens, une philosophie peut être dite réelle parce qu'elle s'applique véridiquement à la réalité (par adaequatio rei et intellectus). Une doctrine dont la fausseté est démontrée quant à son adéquation à la réalité perd en même temps sa propre réalité pour n'être plus qu'une chimère. En ce sens, une doctrine est dite réelle parce qu'elle est vraie (PHP, 98).

Une telle définition, lorsqu'elle est rapportée à la tâche propre de la philosophie, la rend indistincte de celle de la science. Mais contrairement à cette dernière qui prouve sa possibilité par sa réalité même, la métaphysique a été incapable dans son histoire à fournir une vérité probante. La «marche détestable » de la métaphysique permet de douter de sa possibilité même, et par là de sa réalité ${ }^{12}$. Cela n'est vrai, selon Gueroult, que si le philosophe fait dépendre la valeur de la métaphysique de sa capacité à fournir une vérité

12. Gueroult se réfère à l'introduction à la Critique de la raison pure, trad. Jules Barni, Paris, Flammarion, I900, p. 50-5I [B 20-2I; W 59-60]. 
logique et une certitude de même nature que celle des sciences mathématique et physique, et qu'il cherche à savoir comment la métaphysique est possible comme science $(P H P, 67)$. Or pour Gueroult, la métaphysique doit être considérée dans sa réalité de la même façon que la science: «Par la croyance en l'expérience philosophique vivante dans l'histoire, l'attitude du philosophe doit être ici celle de Kant à l'égard de la science de son temps: [...] l'histoire de la philosophie existe en fait, comment est-elle possible?» $(P H P, 43 a)$.

Dans les faits, la réalité des philosophies passées réside avant tout dans «le sentiment de valeur objective que l'historien de la philosophie leur attribue $[. .$.$] à toutes, quelque difficulté qu'on éprouve par ailleurs à déter-$ miner cette valeur et à fonder ce sentiment» (PHP, 65). Gueroult fait donc reposer la condition de possibilité de la dianoématique sur le sentiment de réalité qu'éprouve l'historien de la philosophie au contact de doctrines pourtant contradictoires entre elles ${ }^{13}$ - sans l'expérience vivante subjective, l' "ordre des raisons » d'une doctrine philosophique ne suffit pas à sa réalité. À partir de là, Gueroult cherche une valeur qui soit la même pour la réalité des doctrines et pour la réalité de l'histoire de la philosophie, celle des «conditions de possibilité du sentiment de cette valeur philosophique» qui soutiennent l'expérience philosophique indéfiniment renouvelée dans l'histoire $^{14}($ PHP , 63).

Les philosophies ne peuvent recevoir leur valeur et leur réalité d'un réel commun antérieur à la pensée philosophante puisque si tel était le cas nous constaterions une seule et même théorie ou définition de la réalité; or tandis qu'elles s'estiment toutes qualifiées pour en offrir une, elles sont en fait toutes en désaccord pour dire ce qu'est la réalité (PHP, IOI). Puisque la réalité qui résulte de l'effort philosophant ne peut s'identifier à une réalité première toujours semblable, "toute doctrine retire préalablement au réel donné son titre de réalité, pour ne l'accorder qu'à la représentation d'un réel qu'elle a elle-même fournie» (PHP, IO2-IO3). C'est pourquoi la réalité de la chose à expliquer est engendrée par «l'acte même de la pensée philoso-

13. Pour le sceptique à qui fait défaut le sentiment de cette réalité, il est inutile de chercher à le convaincre du bien-fondé du problème, déclare Gueroult. Tout au plus est-il possible d'objecter l'existence de l'histoire de la philosophie pour convaincre de la réalité du sentiment de réalité.

14. Cette valeur est à la source de la principale objection formulée par les critiques à l'égard de la dianoématique qui réside dans «la prétention à comprendre la plupart des doctrines et le vœu pieux de ne pas s'excepter de leur champ ", prétention à se présenter comme un «système de tous les systèmes " et un "système comme les autres »: Christophe Giolito, "Pratique et fondement de la méthode en histoire de la philosophie chez Martial Gueroult ", Revue de métaphysique et de morale, $\mathrm{n}^{\circ}$ 30, 2001, p. 83 et suivantes. Voir aussi sur ce point Bernard Sève, "La Dianoématique de Martial Gueroult et le problème de l'histoire de la philosophie ", dans Jules Vuillemin (dir.), La philosophie et son histoire. L'âge de la science: lectures philosophiques, Paris, Odile Jacob, I990, p. I67-169; Daniel Parrochia, La raison systématique, Paris, Vrin, I993, p. 29-32. 
phante qui mène l'intellection explicative »; la réalité « résulte à chaque fois d'un jugement thétique» (PHP, 97, 102). De la déduction ressort deux réels: un réel commun et un réel philosophique.

Le réel commun désigne le simple postulat d'une chose à expliquer, indépendante de la pensée philosophante, sur laquelle toute doctrine fonde la validité de sa réalité philosophique propre (postulat réaliste); il est condition de possibilité de toute expérience philosophique vivante dans l'histoire. Le réel commun est cependant entièrement indéterminé, le jugement thétique au fondement de toute doctrine se charge de le produire (postulat idéaliste). Cet acte thétique institue un réel philosophique propre à chaque doctrine et à l'exclusion de celui des autres, mais que chaque doctrine identifie au réel commun. Toute philosophie s'empare donc d'un réel commun à expliquer "pour mettre en doute originellement les déterminations propres à ce "réel commun" [...] et n'affirmer valablement que les déterminations propres au réel philosophique qui leur est particulier» (PHP, I04-I05). L'historien philosophe, privé de la croyance en l'identité du réel philosophique au réel commun, n'en croit pas moins à ce réel commun, condition et source de la réalité des expériences philosophiques, dont la sienne. Cette réalité ne réside pas dans l'objet que chaque philosophie saisit; elle est à la fois intérieure à chaque système, mais commune à tous au titre de condition de possibilité de l'expérience philosophique. En quoi consiste cette réalité ?

Gueroult opère un parallèle entre le sens de la réalité qu'il accorde aux philosophies et les notions de Realität et de Wirklichkeit reprises à Kant, Maïmon et Fichte. Si la vérité se dit d'une représentation qui correspond effectivement au réel, mais que demeure une valeur objective au contenu de la représentation indépendamment d'une correspondance avec un objet, alors la représentation comme idée devient la chose elle-même avec sa réalité - comme Gueroult le reconnaît des idées claires et distinctes, ou des idées adéquates chez Descartes, Spinoza ou Leibniz. Or, lorsqu'un tel concept s'applique au monde de l'expérience dans la mesure où l'expérience en question est rendue possible par ce concept, celui-ci est vrai en plus d'être réel, ainsi des catégories de l'entendement dans la philosophie transcendantale. La Realität désigne la nécessité du concept (généralement obtenue par une démonstration apodictique) rendant possible l'expérience d'un objet; la Wirklichkeit se dit de l'objet qui tire sa réalité de la réalité du concept. Ce type de concept a une Realität sui generis irréductible à la Wirklichkeit de l'objet (PHP, 98-IO०). En ce sens, la démonstration de Gueroult révèle une Realität commune aux philosophies qui rend leur Wirklichkeit possible. Reste à savoir en quoi consiste exactement cette Realität.

À la suite de longues chaînes déductives, Gueroult révèle la finalité de toute philosophie, en quoi consiste sa "réalité suprême» et sa "valeur absolue » : la réalisation de l'autonomie de la volonté. Le réel commun apparaît comme un moyen en vue de cette fin (PHP, 2I8). En tant que condition de possibilité du réel philosophique, le réel commun consiste dans l'identifi- 
cation de l'intérieur et de l'extérieur de la pensée philosophante (PHP, 234). Qu'est-ce à dire?

Pour Gueroult, un système philosophique, dit métaphysique, ne se limite pas à son caractère ontologique; tout système achevé, qui produit un monde à partir de sa compréhension première de l'être, produit en même temps un ordre de valeurs: "Ainsi l'histoire nous fournit, avec l'ensemble des systèmes qui s'y manifestent, tout un clavier de valeurs absolues, comme elle nous fournit tout un clavier de réalités absolues» (PHP, 2 I4). Plus encore, c'est en fonction d'un sentiment subjectif et affectif antérieur au système que nous nous portons vers telle doctrine plutôt que telle autre, et que le philosophe parvient à son propre jugement thétique; celui-ci est le résultat d'une libre préférence, d'une croyance à laquelle chacun pour son propre compte juge impossible de se soustraire ${ }^{15}(P H P, 206)$. Ainsi, le jugement thétique et le jugement de valeur originels qui décrètent une réalité et une valeur absolues sont identiques (PHP, 2I3). La liberté est condition de possibilité de la multiplicité des doctrines données dans l'histoire; sans elle, les réels philosophiques, privés de l'expérience vivante qui leur confère leur caractère pratique, deviendraient choses inertes; les actes spéculatifs originels, en lesquels réside la liberté, n'auraient plus de raison d'être, et la philosophie perdrait sa fonction propre: "La liberté apparaît donc dans chaque système comme consubstantielle à lui. La spéculation est en chacun l'acte même par lequel la liberté se réalise, l'acte où s'identifient spéculation et liberté » (PHP, 215-216).

L'adhésion à une doctrine philosophique relève d'un acte libre que chacun effectue pour soi. L'évidence et les conditions de sa recevabilité que propose chaque doctrine au fondement de sa démonstration ne relèvent pas de la pure théorie. En définitive, l'évidence ne saisit que ceux qui y ont volontairement adhéré, et la nécessité des réels philosophiques repose en leur fondement sur un choix pratique: celui d'un «acte de la volonté libre se voulant elle-même». Se vouloir soi-même en tant que volonté libre est ce qui confère une valeur absolue à chaque réel philosophique. Nous pouvons ainsi comprendre que le réel philosophique de Gueroult s'élabore sur un besoin éminemment pratique du philosophe, autrement dit sur un manque né de l'insatisfaction à agir selon les mœurs et coutumes mal assurées du sens commun ou selon des principes philosophiques inconséquemment justifiés par les doctrines antérieures. Il y a chez Gueroult cette idée propre à une

15. Jacques Bouveresse, qui veut bien accorder ce point à Gueroult, le rapproche de Renouvier: "Appendice I. Martial Gueroult et la philosophie de l'histoire de la philosophie ", en accès libre sur le site du Collège de France, $20 \mathrm{I} 2$, http://books.openedition.org/cdf/I 783 ? lang=fr\#access. Mais l'aspect moral des ontologies ne suffit pas selon Bouveresse à légitimer une "histoire de la vérité » qu'il considère comme contradictoire dans les termes. Il formule le même type de reproche envers Foucault dans Nietzsche contre Foucault, Marseille, Agone, 2016. 
certaine tradition philosophique moderne, de Hegel à Sartre ${ }^{16}$, que les doctrines philosophiques, au moment de leur émergence, totalisent les éléments culturels de leur époque.

Le réel philosophique, fondé sur un réel commun «ontologico-moral», a pour vocation de réaliser l'autonomie de la volonté qui se veut elle-même par la force de contrainte que fournit la logique immanente du système à celui qui a adhéré à son évidence première. La force de contrainte de chaque doctrine est fournie par les déterminations du réel commun ou Realität qui, comme nous l'avons vu, sont également celles d'un réel commun moral, et qui procurent l' "efficacité pratique " (Wirklichkeit) des doctrines métaphysiques, ou «doctrines ontologico-morales", par la force de leur logique contraignante pour la volonté. Ces conditions de possibilités consistent en la synthèse maxima possible des déterminations du monde et la loi de leur synthèse, en l'autosuffisance du système et en l'identité de l'intérieur et de l'extérieur de la pensée philosophante.

L'objet que le philosophe s'attache à expliquer ne s'offre à lui qu'en vue de l'exercice de sa liberté, et "le soi-disant "réel" n'a alors de réalité que dans la mesure où il rend possible l'exercice de la pensée philosophante " (PHP, 24I). Il n'existe pas, chez Gueroult, d'opposition entre l'aspect théorique d'une doctrine et la pratique du philosophe puisque l'élaboration d'un système est l'acte spéculatif nécessaire au philosophe pour penser et déployer sa propre pratique. Tout système philosophique possède donc une valeur à la fois théorique et pratique: "L'acte spéculatif apparaît essentiellement comme acte pratique, et l'acte pratique, essentiellement comme acte spéculatif» (PHP, 237). Le réel philosophique de Gueroult annule l'opposition ressassée entre théorie et pratique; en effet, il ne s'agit pas d'appliquer le réel philosophique dans la réalité puisque c'est l'acte spéculatif qui fournit luimême tout le réel auquel le philosophe est engagé et soumis.

\section{Le réel de la philosophie comme rapport de souveraineté à soi}

Asseoir sa liberté est la raison pour laquelle le philosophe s'engage dans une description du réel. La réalité de la chose à expliquer n'est donc affirmée et justifiée qu'en vertu de cette fin. De façon analogue, nous allons voir que Foucault fait résider le réel de la philosophie dans le rapport de soi à soi.

16. Voir par exemple Jean-Paul Sartre, Critique de la raison dialectique, Paris, Gallimard, I960, p. I5-I6. Pour Gueroult comme pour Sartre, les nouveaux systèmes philosophiques naissent selon les conditions historiques déterminées d'une époque. Les Idées contiennent le principe de tout ce qui conditionne la contingence de l'acte par lequel elles se révèlent à elles-mêmes, autrement dit à la conscience dans l'histoire (PHP, 298). Mais là où les philosophies, une fois apparues à la faveur des déterminations historiques, dépérissent pour Sartre avec le changement de ces conditions, elles demeurent éternelles pour Gueroult à travers leur loi de synthèse et l'identité de l'intérieur et de l'extérieur, que chaque philosophe ultérieur peut réactiver dans son expérience. 
Le réel, dit Foucault dans Subjectivité et vérité, ne peut rendre compte de la raison de l'existence effective des discours sur le réel, autrement dit du réel des discours sur le réel. Comme Gueroult, Foucault dédouble le sens du terme de réel: le réel comme référent du discours, c'est-à-dire la réalité de la chose à expliquer (qui introduit un réel commun indéterminé chez Gueroult); le réel du discours, c'est-à-dire sa raison d'être et sa fonction propre qui lui confère sa réalité à l'intérieur des pratiques humaines (réel philosophique gueroultien qui contient en lui-même ses conditions de possibilités historiques).

Par surcroît, ce que Foucault entend par «le réel», ce sont les pratiques humaines ${ }^{17}$; c'est donc en tant que pratique que le discours possède une réalité. Interroger le réel d'un discours, c'est interroger une pratique discursive dans la fonction qu'elle occupe au sein d'autres pratiques humaines et qui la rend effective (wirklich). Il n'y a donc pas d'un côté la théorie et le discours, de l'autre, la pratique en général, mais des pratiques dont le pluriel, fait bien remarquer Pierre Dardot ${ }^{18}$, est irréductible: "[Ainsi] le discours scientifique consiste lui-même en des pratiques qui, pour être discursives, n'en sont pas moins de véritables pratiques. " Ramener sur le même plan discours théoriques et pratiques humaines "permet d'éviter tout problème d'antériorité de la théorie par rapport à la pratique, et inversement ", disait déjà Foucault dans un entretien de $1966^{19}$. "Plus profondément, conclut Pierre Dardot, c'est tout le problème du rapport de la théorie en général à la pratique en général qui se trouve récusé en tant que problème. » Cependant, reconnaître le caractère pratique des discours ne suffit pas à indiquer en quoi consiste leur réel puisque d'une part, ce réel ne réside pas dans le simple fait de le dire et que, d'autre part, la pratique ne désigne pas simplement ce que font les hommes, mais la manière dont ils le font et l'accompagnent d'un certain discours. Les pratiques sont donc «le lieu d'enchaînement de ce qu'on dit et de ce qu'on fait, des règles qu'on s'impose et des raisons qu'on se donne, des projets et des évidences ${ }^{20}$ ». Le réel du discours n'est donc pas toujours le même, il dépend de la fonction que le discours possède dans son rapport au réel référent dont il parle et aux pratiques qui lui sont connexes: "Il n'y a pas de fonction générale et uniforme; c'est à chaque fois un cas d'espèce» $(S V, 239 a)$. Toute la question sera de savoir si Foucault découvre une fonction générale au discours philosophique, une fonction qui jouerait le rôle de transcendantal.

17. $S V$, p. 247: «Le réel ne fonctionne pas au rationnel. Le réel, du moins si l'on entend par là les pratiques humaines, est toujours dans l'inadéquation, toujours dans le mauvais ajustement. »

18. Pierre Dardot, "De la praxis aux pratiques", dans Marx et Foucault. Lectures, usages, confrontations, Paris, La Découverte, 20I 5 , respectivement p. I 86 et I85.

19. Michel Foucault, "Michel Foucault, Les mots et les choses", Dits et écrits, vol. I, Paris, Gallimard, "Quarto ", 200I, p. 526.

20. Michel Foucault, "Table ronde du 20 mai I 978 ", Dits et écrits, vol. II, p. 84I. 
Enfin, Foucault fait un dernier usage du concept de réel lorsqu'il demande comment s'inscrit le réel de la philosophie dans ce réel qu'est notre histoire $(G S A, 32 \mathrm{I})$; par là, il s'interroge sur l'inscription de la philosophie dans l'histoire des pratiques humaines, alors que l'histoire comme discipline est elle-même une pratique humaine sans laquelle la philosophie dans son histoire ne se serait pas conservée — de façon analogue chez Gueroult, l'histoire de la philosophie est une discipline dans laquelle l'historien fait l'expérience vivante des philosophies passées.

Pour Foucault comme pour Gueroult, il s'agit de partir de la réalité des philosophies, donnée comme un fait, pour en déterminer la raison d'être qui constitue leur réel propre $\left(A S, \mathrm{I}_{5}\right)$. Mais tandis que pour Gueroult les philosophies renferment en elles-mêmes, dans la loi de synthèse et l'identité de l'intérieur et de l'extérieur, les déterminations historiques de leur existence, Foucault cherche dans les conditions historiques la raison d'être, c'est-à-dire le réel propre que les philosophies n'indiquent pas explicitement dans le contenu de leurs énoncés. Puisque pour Gueroult les doctrines contiennent en elles-mêmes les conditions historiques de leur émergence, elles possèdent aussi en elles leur propre raison d'être, et le réel de la pratique philosophique se trouve ainsi dans la doctrine même; chez Foucault, le réel de la philosophie dépend de son inscription au sein des pratiques connexes.

Pour déterminer le réel du discours philosophico-moral de la période hellénistique et romaine à propos du mariage, Foucault introduit les éléments du problème de la façon suivante: premièrement, il existe une littérature moraliste recommandant le mariage et prescrivant un ensemble de conduites précises quant aux rapports entre homme et femme; deuxièmement, la pratique matrimoniale telle que conseillée dans cette littérature s'atteste pour une grande part dans la réalité des pratiques humaines de la période hellénistique et romaine; troisièmement, on ne peut cependant pas considérer que l'effectivité de cette pratique matrimoniale soit l'effet de ces prescriptions, d'abord parce que cette littérature philosophico-morale s'adressait surtout à une élite culturelle tandis que la pratique matrimoniale correspondante est présente dans de nombreux groupes et classes éloignés de cette élite $(S V, 234)$. C'est pourquoi le fait de cette correspondance entre pratique et prescriptions philosophiques est en réalité problématique d'autant plus que Foucault relève l'antériorité de la pratique matrimoniale sur le discours, qui vient la refléter $(S V, 235,245)$. En effet, quel intérêt les philosophes pouvaient-ils trouver à transcrire la réalité sous forme de traités? Si leur raison d'être consiste dans le "redoublement représentatif ", alors ces discours, n'ayant pas d'effets ni de fonction visible, apparaissent comme "discours en trop ». Comme nous l'avons déjà dit, le réel de la chose décrite n'explique pas le réel du discours.

Les traités philosophico-moraux se présentent en fait comme moyens de techniques d'existence (tekhnai peri bion) qui ont pour rôle de "prescrire les procédures de transformation du sujet lui-même [...] qui doivent lui per- 
mettre d'habiter le code, de le pratiquer, de l'accepter " $(S V, 262)$. Du temps de l'organisation politique des cités, le système de valorisation morale de la sexualité était fondé sur le principe d'une activité virile isomorphe aux relations sociales et conjugales - les relations sexuelles s'ajustaient aux rapports sociaux dans un "continuum socio-sexuel». Il se rapportait à une "aristocratie de concurrence ${ }^{21}$ » façonnée par les jeux d'alliances et l'espace agonistique de la cité, dans laquelle l'institution du mariage était "intégrée à titre de pièce parmi d'autres dans le champ des relations sociales", et répondait à des objectifs politiques, économiques et sociaux qui avaient ses raisons dans des systèmes d'alliances $(S V, 260,275)$. Le nouveau système impérial s'organise autour de la cour du souverain et de son système administratif qui définissent la place des individus et restreignent considérablement l'espace de concurrence. L'aristocratie de rivalité laisse place à une aristocratie de service. Le nouveau système politique et administratif de l'Empire amenuise alors les frontières entre les classes: les couches sociales venues "d'en bas" et de la province avec leur mode de vie se mettent à peupler l'administration, à en constituer «les cadres et les rouages"; elles commencent même à investir la cour. Le mouvement initié par les couches populaires « insularise » le mariage, qui devient à la fois hétérogène à toutes les autres relations sociales, et insuperposable à n'importe quelle autre.

Il y a une sorte de "désaphrodisation" du champ social au profit de la relation matrimoniale et d'elle seule [...]. Dans ce nouveau code, les plaisirs majeurs du mariage, ses bénéfices, sont moins liés à l'activité exclusive et totalement dissymétrique du mari qu'aux agréments et avantages d'une vie à deux et d'une certaine réciprocité.

Entre ce nouveau code et l'ancien système de valeurs, il y a incompatibilité. Le système aristocratique de valorisation du mariage attaché au fonctionnement politique des cités perd de sa signification: "de sa valeur économique sans doute, mais surtout de sa valeur politique» $(S V, 260-26 \mathrm{I})$. Cette vieille aristocratie se trouve forcée d'accepter le nouveau modèle de comportement. Ainsi, les discours philosophiques ne s'adressent pas en premier lieu aux couches populaires, mais à cette aristocratie qui, en mal de différenciation reconnaissable, reporte les principes d'activité virile et de maîtrise de soi à l'intérieur de la vie matrimoniale, qui devient le lieu privilégié de leur application ${ }^{22}$. Les discours philosophico-moraux de l'ère impé-

21. Terme qu'il emprunte à Paul Veyne ( $L$ La famille et l'amour sous le Haut-Empire romain ", Annales. Économie, sociétés, civilisations, $\mathrm{n}^{\circ} \mathrm{I}$, I978, p. 35-63).

22. À l'opposé de cette attitude, les élites ont également pu choisir d'accentuer tous les éléments qui manifestent la différence de statut de la façon la plus visible, c'est-à-dire les "marques qui relèvent de l'attitude corporelle, du vêtement et de l'habitat, des gestes de générosité et de magnificence, des conduites de dépense, etc. » La nouvelle organisation du système politique et administratif impérial produit donc de nouvelles manières de se conduire: "Aux nouvelles formes de jeu politique, et aux difficultés de se penser soi-même comme sujet d'acti- 
riale permettent d'ajuster un nouveau code moral à un ancien système de valeurs pour ceux qui les habitent. Le réel de la pratique matrimoniale ainsi décrit par ces discours sert de moyen visant à intensifier le rapport de souveraineté à soi-même: on retrouve ici pour ce qui est des pratiques de soi la manière dont la réalité de l'objet expliqué sert de moyen en vue de l'autonomie de la volonté pour Gueroult.

Si les raisons d'être d'une philosophie sont à chercher du côté des déterminations historiques, Foucault rabat en définitive le réel de la philosophie dans sa pratique historique effective qui consiste dans des techniques de rapport à soi. À la question de savoir comment la philosophie gréco-romaine peut devenir "une activité réelle dans le réel», Foucault répond dans Le souci de soi que "la tâche de [cette] philosophie - son ergon principal et premier - sera précisément d'exercer ce contrôle [des représentations]»:

[à] l'équipement rationnel qui assure une conduite sage [...] s'ajoute la nécessité d'un travail de la pensée sur elle-même [... qui] doit avoir la forme d'un filtrage permanent des représentations: les examiner, les contrôler et les trier. Plus qu'un exercice fait à intervalles réguliers, c'est une attitude constante qu'il faut prendre à l'égard de soi-même ${ }^{23}$.

Le réel de la philosophie réside ici dans une attitude constante à l'égard de soi-même.

D'un autre côté, cette philosophie de la culture de soi s'est élaborée en relation à la nouvelle organisation politique, elle est donc elle-même déjà politique. Selon Lorenzini, seul le cynique, en comparaison des autres philosophes anciens, fait «de la construction éthique de son corps et de sa vie un enjeu qui est en même temps et déjà politique» — bien que, selon lui, toute éthique soit déjà politique quand elle ne concerne pas les philosophes -, car il inscrit son existence scandaleuse au cœur de l'espace public; à l'inverse, le stoïcien se contente d'agir sur ses pensées et son discours intérieur ${ }^{24}$. En réalité, ce n'est pas parce que le stoïcien ne fait pas du "scandale» une finalité que sa philosophie engage une manière de vivre dégagée des liens avec la politique. Tout au contraire, élaborée en relation au champ politique, la philosophie stö̈cienne est aussi déjà politique, quand bien même son ergon réside dans le contrôle de ses représentations.

Quand certains interprètes considèrent le cynisme, qui réduit dans sa pratique le savoir à quelques principes rudimentaires, comme la philosophie que Foucault souhaite réactiver, ils attestent alors que la dimension «archéo-

vité entre une naissance et des fonctions, des pouvoirs et des obligations, des tâches et des droits, des prérogatives et des subordinations, on a pu répondre par une intensification de toutes les marques reconnaissables de statut ou par la recherche d'un rapport adéquat à soimême" (Le souci de soi, Paris, Gallimard, I984, p. II7-I I8).

23. Michel Foucault, Le souci de soi, p. 87-88.

24. Daniele Lorenzini, «Statues visibles de la vérité: l'askêsis corporelle entre éthique et politique ", Dorsal. Revista de Estudios Foucaultianos, n² 2, juin 20I7, p. 45. 
généalogique» de sa philosophie n'est pas ce qui les captive. Ils sous-estiment alors la part fondamentale que constitue la connaissance dans les études que Foucault propose de la philosophie antique. On ne peut pas d'un côté faire usage d'une méthode archéo-généalogique, et de l'autre exhorter à des techniques philosophiques de subjectivation soustraites à l'exigence de vérité ${ }^{25}$, sauf à séparer le travail théorique, que l'on réserve aux philosophes, de l'application pratique, que le philosophe livre sous forme de leçons aux non-philosophes.

\section{Le pragma comme réel de la philosophie}

Durant la période impériale, les philosophes formulent des conseils et des préceptes pour pouvoir se conduire dans la nouvelle organisation politique; le réel de leur philosophie consiste alors dans le rapport à soi qui nourrit cette conduite effective. Platon, pour sa part, semble envisager une relation plus frontale entre philosophie et politique dans la Lettre VII puisqu'il incombe au philosophe d'agir dans le champ politique par l'emploi d'une parole vraie à l'égard du pouvoir. Cependant, dans le commentaire que Foucault propose de cette lettre, le réel de la philosophie ne consiste pas dans un rapport d'efficacité à la politique, auquel cas elle coïnciderait selon lui avec la rhétorique: "L'épreuve du réel qu'est la philosophie, prévient Foucault, ce n'est pas son efficacité politique, c'est le fait qu'elle s'introduit, dans sa différence propre, à l'intérieur du champ politique ${ }^{26} »(G S A$, 2I I-2I2). Dès lors, quelles sont les conditions du réel de la philosophie pour Platon? Et quelle est la différence propre du discours philosophique par rapport à la rhétorique? Foucault repère trois «cercles » où s'atteste le réel de la philosophie: le cercle de l'écoute, le cercle de soi-même, le cercle de la connaissance. Ils sont au principe de la différence propre de la philosophie et de son discours.

25. Comme le propose Lorenzini en conclusion de l'article «La vie comme réel de la philosophie».

26. Lorenzini cite également ce passage et interprète la différence propre du discours philosophique comme l'exercice concret et effectif du dire-vrai face à et par rapport au pouvoir. Deux lignes plus loin, la "question de la réalité » de la philosophie est présentée, non plus comme celle de l'effectivité, mais comme celle de «l'efficacité concrète de son discours " ( La vie comme réel de la philosophie », p. 470-47I). Premièrement, si la différence propre du discours philosophique par rapport aux "autres discours " consiste dans son caractère concret et effectif, cela signifie que les autres discours ne sont ni concrets ni effectifs, or la rhétorique l'est tout autant (Lorenzini semble avoir ici à l'esprit autre chose, une différence avec un discours philosophique qui ne se prononce pas à l'égard du pouvoir). Si, en revanche, la différence propre du discours philosophique est de dire-vrai, contrairement aux autres, on ne constate pas alors de différence entre le discours philosophique et la parrêsia politique, celle de Périclès par exemple. Enfin, si l'exercice concret et effectif du discours philosophique face au pouvoir est synonyme d'efficacité concrète pour Lorenzini, et si nous espérons avoir déjà montré que cette identification était impossible chez Foucault, notons qu'elle contrevient aussi à l'analyse du cercle de l'écoute que nous rapportons plus bas. 
Afin d'observer si la philosophie peut rencontrer son réel dans l'individu, et si cet individu est capable de rencontrer la philosophie dans son réel, il faut, explique Platon, lui montrer pan to pragma (340 b-c), c'est-à-dire lui montrer toute l'affaire de la philosophie, «ce qu'est cette chose, cette chose elle-même ", dit Foucault : "Il faut [lui] montrer pan to pragma: le réel de la philosophie dans son ensemble, tout le réel de la philosophie, tout ce qu'est la philosophie, comme référent à la notion, de la notion de philosophie ». Et il faut lui présenter ce qu'est le pragma de la philosophie, la philosophie dans sa réalité, en lui montrant les pragmata de la philosophie et la peine qu'elle donne. Le pragma, pris en ce second sens, c'est l'activité, ce à quoi on s'occupe et on s'applique; les pragmata, ce sont donc "les affaires, les activités, les difficultés, les pratiques, les exercices, toutes les formes de pratiques auxquelles il faut s'exercer et s'appliquer, et pour lesquelles il faut se donner du mal et qui donnent effectivement du mal» (GSA, 220).

Le passage de la Lettre VII sur le pragma de la philosophie a donné lieu à d'importants débats: pour certains, le pragma désigne l'objet de la philosophie qui ne peut se réduire aux pragmata. Cet objet n'est pas clairement défini dans la lettre mais correspondrait selon certains interprètes aux «réalités suprêmes premières» sur lesquelles Denys aurait écrit un traité (344 b), réalités qui ne peuvent faire l'objet que d'un enseignement oral selon Platon; pour d'autres, pan to pragma renvoie, non pas à un objet de savoir, mais à l'ensemble des activités qui constituent la philosophie ${ }^{27}$. On peut considérer que Foucault se range à cette dernière interprétation quand il traduit pragma, non par "objet» ou "chose », mais par "réel ", réel qu'il indique ensuite se trouver dans les pratiques mêmes de la philosophie, c'està-dire ses pragmata. Et si le réel de la philosophie n'est pas à chercher dans un domaine qui lui est externe, c'est que ce réel porte sur le sujet lui-même: «Ce en quoi la philosophie rencontre son réel, c'est la pratique de la philosophie entendue comme l'ensemble des pratiques par lesquelles le sujet a rapport à lui-même, s'élabore lui-même, travaille sur soi. Le travail de soi sur soi, c'est le réel de la philosophie » $(G S A, 224)$. C'est pourquoi ce réel ne consiste pas seulement dans un discours, une parole vraie face aux puissants, un dialogue ou un enseignement oral, qui sont en fait les moyens

27. Se fondant sur la Lettre VII et le Phèdre, l' «école de Tübingen », autour de Krämer et de Gaiser, a développé à la fin des années I950 l'idée que les dialogues de Platon ne possèdent qu'une valeur protreptique et n'expriment qu'imparfaitement sa philosophie, dont la partie la plus importante portant sur les premiers principes de la réalité ne pouvait être l'objet que d'un enseignement oral à l'intention d'élèves choisis. Voir notamment pour cette thèse Marie-Dominique Richard, L'enseignement oral de Platon, Paris, Cerf, I986, ouvrage préfacé par Pierre Hadot. Luc Brisson, partisan d'un sens de pragma comme activité, avait formulé des objections sur cette tendance au réductionnisme ésotérique: «Présupposés et conséquences d'une interprétation ésotériste de Platon", dans Lectures de Platon, Vrin, 2000, p. 43-I I0; voir également son compte rendu du livre de Richard, "L'enseignement oral de Platon ", Les études philosophiques, Paris, I990, nº I, p. 95-105. 
(comme les tekhnai peri bion par exemple) et en même temps le lieu où se manifeste ce rapport à soi en lequel réside essentiellement le réel de la philosophie. Et parce qu'un rapport à soi n'existe pas en dehors de certaines pratiques, le réel de la philosophie consiste en l'ensemble des pratiques au pluriel par lesquelles un sujet s'élabore soi-même et dans lesquelles il peine, rencontre des difficultés, les surmonte par ses efforts. On peut donc dire que le rapport d'élaboration à soi constitue la finalité de la philosophie, laquelle se manifeste comme réelle à l'endroit de ses pratiques.

L'épreuve du réel de la philosophie que Platon fait passer à Denys consiste à lui montrer le pragma de la philosophie comme une longue route difficile qui met en œuvre un ensemble de pratiques. L'individu qui choisit la philosophie doit alors effectuer une "conversion de la décision " qui consiste en " un choix initial, un cheminement et une application»: "Il s'agit [...] d'un choix qui doit être fait une fois pour toutes et qui doit ensuite se développer, se dérouler et presque se monnayer dans le travail assidu de la vie quotidienne» (GSA, 22I-223). Denys a ostensiblement échoué devant cette épreuve. Dès les premières leçons, il s'est déjà cru en possession des choses les plus importantes de la philosophie, refusant le long chemin qu'elles requièrent. S'il s'est ainsi fourvoyé, c'est parce qu'il a cru que la philosophie se transmettait par mathêmata, par formules apprises par cœur, en dehors d'un difficile rapport à soi; qu'il ait écrit un traité sur «les questions les plus importantes de la philosophie" montre bien qu'il n'a rien compris à ces choses. En effet, le cercle de la connaissance, comme le cercle de soi, consiste en une pratique assidue et continue. Le savoir propre aux choses de la philosophie ne se transmet pas par mathêmata, mais s'acquiert par sunousia peri to pragma, c'est-à-dire par union avec ( «être avec» ou "être-auprèsde») la chose, celle sur laquelle Denys a tenté d'écrire, la chose de la philosophie $^{28}$. La pratique et le réel de la philosophie résident dans cette cohabitation "avec elle », dit Foucault: «la philosophie n'a d'autre réel que ses propres pratiques» (GSA, 228-229).

C'est ici qu'il faut avancer avec prudence, car il ne s'agit pas pour Foucault de dire que le réel de la philosophie réside en dehors de la théorie — avec d'un côté la connaissance, et de l'autre la pratique —, mais que «les pratiques que l'on exerce de soi sur soi » sont "en même temps ces pratiques de connaissance» $(G S A, 236)$. Les pratiques philosophiques ne sont pas indépendantes d'une connaissance ontologique.

Platon explique dans une longue "digression" comment l'impossibilité d'écrire sur la chose de la philosophie est due à un défaut interne du savoir. La connaissance de l'essence, qui ne peut jamais s'énoncer définitivement dans le logos, ne s'obtient que par «frottement» (tribê) de l'âme avec

28. «Il n'y a pas moyen, en effet, de les mettre en formules, comme on fait pour les autres sciences, mais c'est quand on a longtemps fréquenté ces problèmes, quand on a vécu avec eux... ", 34 I c-d, traduction de Joseph Souilhé. 
les différents éléments de la connaissance, ce à quoi ne peut répondre l'écriture qui fixe la connaissance en mathêma. Cependant, il ne faut pas comprendre que Platon valorise le logos oral par rapport à l'écrit qui est condamné - comme l'entendent Hadot et Lorenzini —, les deux formes sont en réalité rejetées en tant que logos, ainsi que l'observe Foucault (GSA, 234-235); simplement, en tant que moyen, Platon fait ailleurs valoir le dialogue comme meilleur et moins dangereux que l'écrit ${ }^{29}$. Foucault indique que tribê signifie également dans un sens plus général «tout ce qui est exercice, tout ce qui est entraînement [...,] ce par quoi on s'habitue, on s'exerce à quelque chose "; il peut alors conclure que "cette connaissance dernière ne s'obtient et ne s'acquiert que par [...] une pratique continuelle, par une pratique continuellement exercée, par une pratique de frottement entre les autres modes de connaissance» $(G S A, 232)$.

On accède à la connaissance de la chose elle-même "par le va-et-vient, la montée et la descente le long des quatre autres degrés de connaissance [le nom, la définition, l'image et l'epistemê qui est à la fois orthê doxa et nô̂s] et à travers les instruments qui caractérisent les autres formes de connaissance "; mais encore faut-il que l'âme soit de bonne qualité, qu'elle soit suggenês, c'est-à-dire en affinité "avec la chose même, to pragma justement » (GSA, 230-232; Foucault fait référence à 344 a). C'est ce long et dur travail de montée et de descente effectué par l'âme de bonne qualité le long des autres formes de connaissance pour accéder à la réalité dans son être même qui est désigné par le terme de tribê. La connaissance proprement philosophique, autrement dit la philosophie non comme logos mais bien comme ergon, est parfaitement différente des quatre autres formes de la connaissance puisqu'elle consiste en une pratique assidue et permanente, bien qu'elle réside en effet dans «le frottement continu des modes de

29. Le refus platonicien de l'écriture est certainement la thèse la plus commentée de cette lettre VII — thèse qui apparaît également dans le Phèdre. S'il était possible d'exposer dans des écrits ce en quoi consistent les choses les plus hautes de la philosophie, ces textes seraient inutiles ou bien dangereux, écrit Platon. Foucault commente alors ce passage en le rapportant au réel de la philosophie: "Ce serait dangereux pour ceux qui effectivement, ne sachant pas que la philosophie n'a d'autre réel que ses propres pratiques, ceux-là croiraient connaître la philosophie, en tireraient vanité, suffisance et mépris pour les autres, et donc ce serait dangereux. Quant aux autres, à ceux qui savent parfaitement que le réel de la philosophie est dans cette, dans sa et dans ces pratiques, [...] ceux-là n'ont pas besoin de cet enseignement explicite sous forme des mathêmata. Il leur suffit d'une endeixis: d'une indication» (GSA, 229-230). Ceux-là, sur "quelques indications", savent eux-mêmes découvrir la vérité (34I e). Si l'on considère d'une part que les pratiques liées au christianisme ont fait de la parole le lieu privilégié de l'aveu, d'autre part que le développement de l'exégèse a créé un rapport spécifique du sujet connaissant au texte, enfin que la philosophie moderne s'est développée en même temps que l'édition et la presse, peut-être peut-on faire l'hypothèse que la philosophie occidentale a transféré dans le rapport à l'écrit les vertus que Platon reconnaissait à la pratique du discours oral — vertus que l'on reconnaît par exemple dans l'exercice universitaire de l'explication de texte qui consiste à manifester le non-dit de ce qui est écrit. 
connaissance les uns avec les autres ». La notion de tribê permet à Foucault de conclure à un certain avènement de la philosophie en Occident, celui «d'une philosophie dans laquelle le réel même de la philosophie serait la pratique de soi sur soi ", et qui est à mettre en jeu dans le quotidien de l'existence. La notion de tribê s'oppose donc à la figure du sage pleinement possesseur d'un logos d'où découle sa vertu, et que le quotidien des pratiques qui mettent en jeu le savoir ne peut pas venir ébranler ou modifier. Le philosophe, lui, ne met pas simplement la connaissance au service de la vie, il fait de la vie un chemin de connaissance.

Si le réel de la philosophie s'atteste dans l'affinité de l'âme avec le pragma, "la chose même", celle-ci désigne-t-elle pour Foucault, dans une forme tautologique, l'ensemble des pratiques philosophiques - comme il l'a établi lorsqu'il a référé le pragma aux pragmata? Il faudrait alors dire comme Pierre Hadot que le savoir jaillit dans l'âme «lorsqu'on a eu une longue familiarité avec l'activité en quoi il consiste et qu'on y a consacré sa vie ${ }^{30} »$. Ou bien, cette occurrence du pragma fait-elle référence au cinquième degré de la connaissance, au to on. Ce passage de la Lettre VII ( 344 a) a été un autre terrain du débat concernant l'existence d'une doctrine ésotérique platonicienne. De son côté, Foucault dit simplement que la pratique assidue (tribê) des modes de connaissance met «en présence de la réalité de l'Être même» (GSA, 235236), sans s'avancer sur cette "chose-même" avec quoi l'âme doit être en affinité. Le pragma désigne ici pour Foucault la réalité de l'être, l'affinité avec le $t o \mathrm{O}^{31}$; et la pratique par laquelle le sujet est mis en présence de la réalité de l'être répond à la définition que Foucault donnait de la spiritualité dans le cours de l'année précédente: pratiques, exercices par lesquels le sujet s'élabore lui-même pour accéder à la vérité de l'être ${ }^{32}$. Ainsi Foucault, dans l'introduction à L'usage des plaisirs, propose de lire sa philosophie, c'est-à-dire ses écrits, à la fois comme des essais et comme des ontologies.

30. Pierre Hadot, Qu'est-ce que la philosophie antique?, Paris, Gallimard, «Folio essais ", I995, p. I I 5. Luc Brisson réfère également cette occurrence de pragma à l'activité.

31. Notons que ce passage de la Lettre VII est assez proche de celui de la Rhétorique d'Aristote commenté par Pierre Hadot dans son article qui recense les différents sens du mot pragma dans la tradition philosophique grecque: "Il ne faut pas oublier que toutes les considérations de ce genre [dans les prologues] sont étrangères au discours (logos). Car elles ne visent que l'auditeur de basse qualité, qui ne prête l'oreille qu'à ce qui est en dehors du point à discuter (pragma); si en effet l'auditeur n'est pas de cette basse qualité, l'on n'a pas besoin d'exorde, sauf ce qu'il en faut pour exposer sommairement le fait qui est en question (pragma). " Pragma signifie ici "ce dont on parle", "le sujet en question ». Les auditeurs de basse qualité ont besoin de prologues pour saisir le pragma, car ils ne cessent pas de prendre en considération des éléments extérieurs à ce dont il est question. Ce sens de pragma, rapporté au passage de la Lettre VII, signifie que l'âme de bien des hommes n'est pas capable d'être "en affinité » avec «le sujet dont il est question" dans les discussions. Pierre Hadot, "Sur divers sens du mot pragma dans la tradition philosophique grecque", dans Études de philosophie ancienne, Paris, Les Belles Lettres, 20IO, p. 62; nous citons Aristote, Rhétorique, III, I4, I4I 5 b 4, dans ce qui semble être la propre traduction de Pierre Hadot (il souligne).

32. Michel Foucault, L’herméneutique du sujet, Paris, Gallimard-Seuil, 200I, p. I9-20. 
Pour Platon, l'être de la chose ne peut être atteint que par une pratique assidue du raisonnement qui façonne l'âme selon de bonnes dispositions; celles-ci doivent cependant exister en germe dans l'âme pour pouvoir se développer et, à leur tour, former un sens aiguisé du raisonnement. Celui qui a cultivé ces qualités par la tribê n'a besoin que de quelques indications pour parvenir à la vérité sur le sujet dont il est question. La philosophie ne s'enseigne donc ni dans des formules écrites ni dans des discours oraux (34I d-e), mais seulement "par elle-même" et dans des «discussions paisibles".

Foucault repère dans le Gorgias en quoi consistent ces «discussions paisibles» et les bonnes dispositions dont le sujet doit faire preuve dans l'usage du logos pour accéder à la vérité, et ne pas tomber dans le langage de la rhétorique. Dans le jeu dialectique, l'individu ne doit pas dire autre chose que ce qu'il sait être vrai; il doit posséder un sentiment de bienveillance relevant de l'amitié pour le partenaire avec qui il effectue la recherche de la vérité; il ne doit pas avoir peur de dire ce qu'il sait par honte ou timidité (GSA, 339-342). Pour manifester son réel dans sa différence propre par rapport à la rhétorique, ce logos, dit Foucault, doit être un logos etumos, c'est-à-dire un logos authentique tel qu'il est défini par Platon, mais plus largement par la conception grecque du langage: «Le langage, les mots, les phrases, dans leur réalité même, ont un rapport originaire à la vérité. Le langage, les mots, les phrases portent avec eux ce qui est la vérité » (GSA, 290). Pourtant, et bien que le logos ait un rapport originaire à la vérité pour Platon, il ne pourra jamais la livrer de manière définitive. Gueroult cherchait la différence propre de la philosophie à l'égard de la science; chez Platon, le discours philosophique exerce sa fonction propre en se distinguant de la rhétorique, évitant tout ornement et toute construction qui vise à produire de l'effet sur l'autre. Il doit se présenter comme un langage à l'état nu, «à ce point dépouillé et simple » qu'il sera véritablement adéquat à ce à quoi il se réfère. Mais il sera dans le même temps conforme au mouvement même de la pensée et fidèle à ce que croit le locuteur. Il y a un "acte de confiance " et de foi (pistis) «entre lui-même et ce qu'il dit». S'il est authentique, le logos "dira le vrai du réel et il dira aussi ce qu'est l'âme de celui qui le prononce » (GSA, 289-290). Le discours philosophique est donc toujours, pour celui qui le prononce, "aussi proche que possible du réel auquel il se réfère" (GSA, 300).

Dans le logos, le réel de la chose à comprendre apparaît toujours équivoque car, dans le domaine de la rhétorique, il est toujours possible selon Platon de réfuter celui qui raisonne sur le cinquième élément de la connaissance $(343$ d). Le réel de la chose, apparaissant à l'âme dans la tribê, se confond alors avec cette pratique même qui met en présence de la réalité de l'être, sans qu'il soit possible d'affirmer l'antériorité ou l'extériorité de l'être par rapport à l'activité circulaire de la connaissance. Que celle-ci mette simplement en présence de l'Être (Platon) ou bien qu'elle produise sa réalité (Gueroult), c'est à chaque fois la difficile activité philosophique - et non le 
logos en son contenu — qui offre en son cours la «révélation de l'être». Si le doute radical est chez Gueroult l'opérateur initial qui permet l'acte conceptuel de fondation et d'élaboration de l'ontologie, on peut dire que chez Foucault - dans le commentaire de la Lettre VII, mais aussi dans sa propre pensée - le doute est un élément permanent de sa pratique philosophique et du rapport à l'ontologie — c'est pourquoi il peut envisager ses écrits comme des "essais ${ }^{33}$ ». Chez Platon, parce que le logos seul ne suffit pas à délivrer la connaissance de l'être sans l'activité du sujet de «bonne qualité », que la connaissance de l'être n'est jamais acquise par avance par la connaissance des mathêmata, ni par la connaissance du nom, de l'image, de la définition, de la science ou de l'opinion droite, le pragma renvoie à la fois à la connaissance du sujet en question ( «la chose dont il s'agit») et à l'activité de connaissance qui délivre la chose.

Le défaut interne de la connaissance par le moyen du seul logos donne place à la tribê qui ne peut délivrer un savoir et une vertu définitifs et ouvre à l'infinité de la spéculation. De son côté, Gueroult déduisait la liberté infinie de la spéculation de la limite de chaque réel philosophique à son monde propre $(P H P, 223)$, ouvrant ainsi les réels philosophiques à un nombre possiblement infini. En revanche, en ce qui concerne le rapport quotidien du philosophe à "sa" doctrine, Gueroult semble considérer que les actes pratiques de la philosophie, identifiés aux actes spéculatifs originaires, dictent sans aucun reste la conduite à tenir. Dans cette mesure, la réalisation de l'autonomie par le philosophe s'apparente chez Gueroult à celle du sage, quand chez Platon l'affinité avec la chose de la philosophie ne délivre pas un savoir et une vertu définitifs.

Enfin, qu'en est-il du réel de la philosophie dans son rapport à la politique? Dans la Lettre VII, ce réel réside dans le cercle de l'écoute. Pour que le discours philosophique en tant que pratique de véridiction rencontre son réel, il faut que ceux auxquels il s'adresse veuillent écouter et suivre les conseils qui seront donnés; sinon le philosophe doit «faire comme les médecins qui s'en vont lorsque les clients et les malades ne veulent pas écouter leurs prescriptions» (GSA, 2I3). Par conséquent, la philosophie dans son effectivité suppose la volonté de bonne qualité de l'auditeur avec laquelle elle puisse être en affinité; autrement dit, le réel de la philosophie suppose la volonté philosophique de ceux qui veulent écouter, ce en quoi consiste le cercle de l'écoute. Dans le champ de la politique, il faut que ceux qui ont à gouverner (monarques, aristocrates, citoyens, etc.) possèdent une attente et une écoute à l'égard du discours du philosophe pour informer leur volonté politique. L'ergon de la philosophie dans le champ de la politique réside dans l'articulation du gouvernement de soi et du gouvernement des autres $(G S A, 236)$, et il ne repose pas ailleurs que dans le rapport de soi à soi de celui qui a à gouverner. Et si la philosophie ne peut jamais prendre la forme 
des mathêmata, la philosophie ne donnera jamais par avance les lois à ceux qui ont à gouverner ${ }^{34}$. Il est en revanche nécessaire que celui qui a à gouverner pratique en même temps la philosophie pour que se manifeste son ergon.

\section{Conclusion}

Il ne s'agissait pas pour Foucault de demander à Platon et aux philosophes antiques ce qu'il faudrait faire pour que la philosophie soit politiquement (plus) efficace; il s'agissait de voir en quoi consistait le réel de la philosophie antique afin d'offrir une nouvelle perspective sur le réel de la philosophie moderne. Le réel de la philosophie, en tant que travail difficile du sujet sur lui-même, a pour condition une pratique de véridiction à propos du réel; il a également pour condition un rapport du sujet au champ politique; la philosophie a enfin pour condition de réalité un rapport du sujet à un système de valeurs morales donné par une culture ${ }^{35}$. Dans son étude, Foucault ne présente pas deux réels de la philosophie - une confrontation au pouvoir et une pratique continue de l'âme $\mathrm{e}^{36}$-, mais un réel (dans le rapport laborieux de soi à soi) avec trois conditions de réalité (vérité, éthique, politique), c'està-dire trois champs qui sont fonctions de la raison d'être de la philosophie au sein des pratiques humaines et qui permettent de l'inscrire dans le réel de notre histoire. Ainsi, en ce qui concerne la philosophie, l'a priori historique ne désigne plus l'ensemble des règles qui caractérisent une pratique, mais l'ensemble des fonctions qu'une pratique réalise à l'égard d'autres pratiques qui lui sont corrélées.

$\mathrm{Si}$, pour Gueroult, une doctrine philosophique est le moyen par lequel un individu cherche à établir (ou rétablir) un équilibre avec son milieu quand ce rapport est troublé par l'inquiétude et l'insatisfaction ressenties à l'égard de la, ou des morales données présentement par ce milieu, Foucault décrit de façon analogue la pensée de Canguilhem à l'égard des sciences de la vie:

Que l'homme vive dans un milieu conceptuellement architecturé ne prouve pas qu'il s'est détourné de la vie par quelque oubli ou qu'un drame historique l'en a séparé; mais seulement qu'il vit d'une certaine manière, qu'il a, avec son milieu, un rapport tel qu'il n'a pas sur lui un point de vue fixe, qu'il est mobile

34. C'est pourquoi nous ne pouvons pas suivre Lorenzini lorsqu'il pense que Foucault est en voie de se rapprocher des cyniques dans cette analyse parce que ceux-là, comme Platon dans la Lettre VII, appliquent "concrètement à la vie quotidienne" «ce que tous les autres philosophes admettent en théorie» ("La vie comme réel de la philosophie», art. cité, p. 477). Que ce soit pour une législation ou pour l'éthique individuelle, il n'existe pas de principes théoriques admis d'avance à appliquer à la cité ou à la vie quotidienne.

35. On retrouve par exemple de façon étonnante tous les traits du réel de la philosophie de la Lettre VII dans la leçon inaugurale de Schelling à l'Université de Berlin en I84I. Voir son "Discours d'ouverture", dans Système de l'idéalisme transcendantal, Paul Grimblot, trad., Paris, Librairie philosophique de Ladrange, I 842, p. 405-4I6.

36. Comme le propose Frédéric Gros, «Résumé », GSA, p. 354. 
sur un territoire indéfini ou assez largement défini, qu'il a à mouvoir les choses les unes par rapport aux autres pour les rendre utiles. Former des concepts, c'est une manière de vivre et non de tuer la vie; c'est une façon de vivre dans une relative mobilité et non pas une tentative pour immobiliser la vie; c'est manifester, parmi ces milliards de vivants qui informent leur milieu et s'informent à partir de lui, une innovation qu'on jugera comme on voudra, infime ou considérable: un type bien particulier d'information ${ }^{37}$.

Dernier texte pour lequel Foucault donna son accord pour publication. Il y a certainement, dans ces quelques lignes sur Canguilhem, des éléments qui nous mettent en garde contre une volonté de surestimer la place de la philosophie cynique dans les derniers cours de Foucault. Que le philosophe cherche à "théoriser» son milieu ne signifie pas qu'il s'est détourné de la vie et qu'il devrait plutôt se livrer à la philosophie comme manière de vivre, prévient "Foucault-Canguilhem»; tout au contraire, former des concepts est, pour lui, une manière de vivre.

37. «La vie: l'expérience et la science» [1985], Dits et écrits, t. II, Paris, Gallimard, «Quarto", p. I 593. 